\title{
Response to Letter to the Editor
}

CARTILAGE

2019, Vol. 10(4) 506-507

(C) The Author(s) 2019

Article reuse guidelines:

sagepub.com/journals-permissions

DOI: $10.1177 / 1947603519861752$

journals.sagepub.com/home/CAR

@SAGE

\section{Dear Editor,}

We thank the authors for their letter and find the authors' comments to be both valid and highly representative of the challenges when designing quality randomized controlled trials (RCTs) to investigate cell therapies for orthopedic or other musculoskeletal pain conditions.

We first respond to the authors' accurately referenced timeline of 2 publications resulting from this trial. The reasons are 2-fold. The initial 2017 publication is mostly a technique and product characterization paper. ${ }^{1}$ Because of previous bone marrow aspirate concentrate (BMAC) publications' inadequate details of cell products used and technique employed, orthobiologics experts now agree upon Minimum Information for Studies Evaluating Biologics in Orthopedics (MIBO), which specifically relate to the use of mesenchymal stem cell (MSC) products (such as BMAC). ${ }^{2}$ Given that we complied with Food and Drug Administration (FDA) and the International Society for Cellular Therapy (ISCT) recommendations for cell characterization via flow cytometry, we wanted to draw attention to our technique and the specific resulting product. However, at the time of publication, escalating unsubstantiated clinical use of BMAC and some misleading claims about cell therapy for arthritis motivated our decision to include early outcomes that were felt to be valuable data for rapid publication, given no RCTs had yet been published at that time. We also felt, a single publication reporting thorough technique description, product characterization, analysis of magnetic resonance imaging (MRI) data and all patient-reported outcomes results, with full discussion of each of these would have made for a lengthy manuscript and subsequently outside the word count guidelines for publication in many orthopedic journals. ${ }^{3}$ Such a conundrum has recently been lamented by journal editors in the field. ${ }^{4}$ It is likely for the foreseeable future that cell therapy trials in orthopedics will remain a challenge to publish complete study results alongside necessary manufacturing description (MIBO) in single manuscripts containing all the information valuable to the reader under current journal word count limits.

Second, we agree that it would have been ideal to have reported 3-year follow-up data. We did track patient safety information for 2 years at the request of FDA, however, due to funding constraints, MRI and patient-reported outcome measures were limited to 1 year for our study. We hope that future studies are able to assess longer-term outcomes related to treatment of knee osteoarthritis using BMAC.

Third, we agree that it is unfortunate that the randomization process yielded assignments to BMAC intervention in greater numbers of knees with prior arthroscopy than not. It is certainly possible that prior arthroscopy could have negatively affected the BMAC outcome, however based on the descriptive summaries presented for the subset of 14 patients without prior arthroscopy, results were relatively similar to those of the overall cohort (Supplemental Tables 1-3). Similarly, the assignment of more Kellgren-Lawrence (KL) grade 3 knees to BMAC than to saline may also have negatively affected outcomes. However, in analysis of only the 11 patients with a KL grade of 1 or 2 on both knees, results were again relatively similar to those of the overall patient cohort (Supplemental Tables 1-3). Although increasing the difficulty of patient accrual, larger definitive RCTs that utilize a paired design should match knees within patient according to both prior arthroscopy and KL grade in order to avoid confounding by these measures.

Fourth, regarding the lack of use of the International Knee Documentation Committee (IKDC) and the Western Ontario and McMaster Universities Osteoarthritis Index (WOMAC) scores, we would like to point out that the Osteoarthritis Research Society International-Intermittent and Constant Osteoarthritis Pain (OARSI-ICOAP) tool has been validated against Knee Injury and Osteoarthritis Outcome Score (KOOS) and WOMAC (more traditional PROMs) and is developed by the OARSI. In addition to measuring pain, the tool makes the distinction between constant pain and pain that comes and goes. This distinction has been shown to be an important one to the patient perspective and highly relevant to pain from knee OA such that we are hopeful more studies will make use of the ICOAP in the future.

Fifth, regarding the addition of platelet-poor plasma (PPP) as a "vehicle" for the BMAC, we were aware that this would be somewhat controversial. During protocol development and the FDA Investigational New Drug application process in 2012, no clear protocol for use of orthobiologics in the knee existed, and thus our treatment protocol was influenced heavily by early proposed mechanisms of therapeutic action from in vitro and in vivo studies of orthobiologics like platelet-rich plasma (PRP) 
demonstrating release of biologic factors fairly quickly on contact of target tissue. Given that the knee joint can hold large amounts of joint fluid, our intent was to coat as much as possible of the cartilaginous and intrasynovial surface with BMAC, avoiding the potential of a too small volume BMAC sequestered in a joint recess, possibly forming a clot, and thus not contacting the needed target tissue and exerting its full biologic therapeutic effect. We agree that PPP may have valuable biologic potential itself and recognize this as a confounder, but would point out that in the absence of a universally agreed-upon BMAC protocol, ours simply represents one of several not yet validated orthobiologic interventions for knee arthritis. Additionally, even if this technique were found to be suboptimal, within the manufacturing regulatory confines of the FDA Investigational New Drug, this was largely unchangeable once we were locked into the initial protocol design process.

Sixth, we appreciate the authors' comments regarding the use of the patients' own knees as a control. Although the use of such a study design does have significant advantages in comparison to a parallel groups design where each patient receives only 1 of the 2 treatments (e.g., completely eliminating patient-level confounding factors, higher power to detect differences at a given sample size), at the time of study design, we were aware that this is an imperfect way to control for pain. Nevertheless, it was a compromise we felt reasonable to make given the difficulty in patient recruitment for a study employing a sham bone marrow aspiration (and no other way to blind patients to treatment allocation). Given what we learned about outcomes, we would not undertake future studies with a similar study design, and in fact have designed a follow-up clinical trial that utilizes an alternative blinding strategy storing BMAC for future use (ClinicalTrials.gov Identifier: NCT03271229).

Finally, the authors' expressed concern that the statistical analysis did not include a "correction for patient" and suggest that a generalized linear model including patient as a random effect could have been employed to accomplish this. However, the paired Wilcoxon signed rank tests that were used did actually correct for patient, as within-patient differences in outcome measures are utilized in these tests, thus accounting for the paired nature of the data.

In conclusion, we greatly appreciate the comments made by the authors, and hope that our study in combination with the concerns highlighted by the authors will form the basis for a future robust larger randomized trial evaluating the efficacy of BMAC in treatment osteoarthritis of the knee which we can agree is unquestionably needed.

\section{References}

1. Shapiro SA, Kazmerchak SE, Heckman MG, Zubair AC, O'Connor MI. A prospective, single-blind, placebocontrolled trial of bone marrow aspirate concentrate for knee osteoarthritis. Am J Sports Med. 2017;45(1):82-90. doi:10.1177/0363546516662455

2. Murray IR, Geeslin AG, Goudie EB, Petrigliano FA, LaPrade RF. Minimum Information for Studies Evaluating Biologics in Orthopaedics (MIBO): platelet-rich plasma and mesenchymal stem cells. J Bone Joint Surg Am. 2017;99(10):809-19. doi:10.2106/JBJS.16.00793

3. Shapiro SA, Arthurs JR, Heckman MG, Bestic JM, Kazmerchak SE, Diehl NN, et al. Quantitative T2 MRI mapping and 12-month follow-up in a randomized, blinded, placebo controlled trial of bone marrow aspiration and concentration for osteoarthritis of the knees. Cartilage. Epub 2018 August 30. doi:10.1177/1947603518796142

4. Piuzzi NS, Dominici M, Long M, Pascual-Garrido C. Proceedings of the signature series symposium "cellular therapies for orthopaedics and musculoskeletal disease proven and unproven therapies - promise, facts and fantasy," International Society for Cellular Therapies, Montreal, Canada, May 2, 2018. Cytotherapy. 2018;20(11):1381-1400.

Shane A. Shapiro Jennifer R. Arthurs Michael G. Heckman Joseph M. Bestic Nancy N. Diehl Abba C. Zubair Mayo Clinic, Jacksonville, FL, USA Mary I. O'Connor Yale University School of Medicine, CT, USA 